\title{
Alter
}

Revue de phénoménologie

$20 \mid 2012$

Eros

\section{D’un phénomène érotique}

\author{
Jean-Luc Marion
}

\section{OpenEdition}

\section{Journals}

Édition électronique

URL : http://journals.openedition.org/alter/1073

DOI : 10.4000/alter.1073

ISSN : 2558-7927

\section{Éditeur :}

Association ALTER, Archives Husserl (CNRS-UMR 8547)

\section{Édition imprimée}

Date de publication : 1 décembre 2012

Pagination : 129-142

ISBN : 978-2-95-223748-2

ISSN : 1249-8947

\section{Référence électronique}

Jean-Luc Marion, « D’un phénomène érotique », Alter [En ligne], 20 | 2012, mis en ligne le 01 juillet 2019, consulté le 28 juillet 2019. URL : http://journals.openedition.org/alter/1073 ; DOI : 10.4000/ alter.1073 


\title{
D’UN PHÉNOMÈNE ÉROTIQUE
}

\author{
Jean-Luc Marion
}

Texte établi à partir d'une transcription par Yves Millou (Université de Rouen), revue et corrigée par Laurent Perreau (Université de Picardie Jules Verne).

Je suis heureux que cette petite conférence s'inscrive dans le programme plus large d'un colloque consacré à la question de l'Eros, sujet dont je voudrais précisément, ici, montrer la centralité. En effet la question de l'Eros devrait apparaître comme centrale, bien que ou plutôt parce que tel n'est pas toujours le cas en philosophie, du moins en philosophie moderne. Nietzsche a pu dire, dans un fragment de 1886 : "Je n'ai jamais blasphémé le nom saint de l'amour - ich habe den heiligen Namen der Liebe entweiht $»^{1}$ - je ne l'ai jamais désacralisé, pourrait-on aussi traduire. D'une certaine façon, on ne peut pas dire que la philosophie soit restée, sur ce point, dans la ligne de la piété de Nietzsche. Il y a une façon, en effet, de désacraliser la question de l'amour. Elle consiste toujours, en dernière analyse, à reconduire, autrement dit à réduire, l'amour à une instance différente de luimême. Les manières d'opérer cette réduction peuvent différer, mais le résultat reste toujours identique, à savoir que l'amour doit se trouver élevé au concept. Et ici s'impose une très célèbre phrase de Hegel, dans la Préface à la Phénoménologie de l'Esprit, qui devrait nous frapper et il est intéressant de constater qu'elle ne nous frappe peutêtre pas autant qu'il conviendrait. Je traduis Hegel : « La vie de Dieu et de l'intelligence divine, doit, si l'on veut, être exposée comme l'amour qui joue avec lui-même. Mais cette idée tombe dans l'édification, et même dans la fadeur, s'il lui manque le sérieux, la souffrance, la patience, et le travail du négatif, der Ernst, der Schmerz, die

${ }^{1}$ Fragments posthumes, 1 [216], Werke, Berlin, éd. Colli \& Montinari, Bd. VIII/1, 1974, p. 54. 
Geduld und Arbeit des Negativen $»^{2}$. Cette phrase sonne de manière tout à fait extraordinaire. Elle est extraordinaire puisqu'elle suppose que, bien entendu, s'il s'agit de parler de l'intelligence divine et de la vie de Dieu, l'amour constituerait certes le lexique approprié, mais que pourtant ce lexique laissé à lui-même sombre dans «...l'édification et la fadeur » - discours purement rhétorique d'un sermon bien-pensant - parce que lui manque le propre du négatif, c'est-à-dire, pour Hegel, du concept : le concept seul devient sérieux, parce que lui seul sait souffrir, sait endurer sa souffrance et en fait son travail, alors que l'amour non seulement manque de la rationalité du concept, mais surtout du sérieux de la souffrance. Alors cette formulation est tout à fait étonnante, parce que d'une certaine façon - et il n'est pas besoin d'être ici un lecteur attentif de Kierkegaard et de ce que Kierkegaard appelait les travaux de l'amour - pour comprendre que l'une des caractéristiques de l'amour, c'est qu'il connait la patience de la souffrance et le sérieux du travail. Comment Hegel a-t-il pu laisser supposer que le concept en savait plus que l'amour à propos de la douleur, du sérieux, de la patience et du travail du négatif ? Si l'on regarde Kierkegaard, lorsqu'il parle, contre Hegel, des œuvres de l'amour, on constate qu'il se réfère évidemment à Saint Paul, exposant que l'amour (ici nommé agapè) déploie justement l'équivalent des quatre caractères prêtés par Hegel au négatif : « La charité endure tout (stregei, Ernst), elle croit tout (pisteuei, Schmerz), elle espère tout (elpizei, Arbeit?) et supporte tout (upomenei, Geduld)»(Corinthiens 13, 7). Comment la philosophie a-t-elle pu tenir pour allant de soi que l'amour ne sache rien de ce que c'est que la souffrance, la patience, la douleur du négatif, en sorte qu'il faille passer au concept pour les rencontrer? Comment peut-on sérieusement méconnaître que l'amour, Eros ou agapè peu importe ici, n'ait aucune expérience de ces dimensions du négatif et que, plus étrange encore, ce soit le concept, dans l'abstraction de la théorie, qui en porte témoignage? Mais, plus étonnant encore, comment se fait-il que la philosophie, sous la figure de Hegel - car, après tout, Hegel peut bien parler au nom de toute la philosophie, qu'il prétend avoir accomplie - ait l'audace de présupposer comme une évidence dispensée de toute démonstration que lorsqu'il y a de l'amour, il n'y a pas de concept ? Va-t-il à ce point de soi que l'amour reste au pied du concept et que le concept seul sache ce que le négatif implique de sérieux, de travail, de patience et de douleur?

2 Phänomenologie des Geistes, Hambourg, éd. J. Hoffmeister, 1952, p. 20. 
Face à ces intenables prétentions, je soutiendrai la possibilité de l'approche d'une pensée argumentée, rationnelle et donc conceptuelle de l'amour; ce qui présuppose de poser la question de savoir si l'amour peut s'élaborer lui-même, si je puis m'exprimer ainsi, son propre concept plutôt que de recevoir sa détermination conceptuelle d'ailleurs. Recevoir sa détermination conceptuelle d'ailleurs semble en effet le sort que la philosophie moderne a, dans le meilleur des cas (celui où elle lui en concédait un, de concept), réservé à l'amour. Comme je l'ai indiqué assez souvent ailleurs, on peut dire que, dans la philosophie moderne pour une large part, l'amour ne brille que par son absence, ou ce qui revient au même, par l'absence du concept en lui. Un indice, sans aucun doute très significatif, a été fourni par la traduction française de Descartes. Lorsque les Meditationes de prima Philosophia définisse ce qu'est le «je » qui pense, à savoir une chose pensante, elles le définissent comme une chose qui pense, c'est-à-dire "dubitans, affirmans, negans, parva intelligans, multa ignorans, volans, imaginans quoque, et sentians $»^{3}$, ce qui se traduit naturellement « une chose qui pense, c'est à dire qui doute, qui affirme, qui nie, qui connait peu de choses, en ignore beaucoup, qui veut, qui imagine aussi, et qui sent». Or il est remarquable que le Duc de Luynes, traducteur français, complète cette liste de modalités de la chose pensante sans qu'on ne lui demande rien, en interposant, au milieu de la séquence, "qui aime et qui hait $»^{4}$. Cet ajout capital fait apparaître que Descartes n'avait pas compté l'amour et la haine parmi les modalités primitives de la res cogitans; autrement dit, la chose qui pense, l'ego du cogito, n'a pas pour Descartes la détermination fondamentale d'aimer. Et ceci constitue un bon indice de la question qui nous occupe : pour construire le concept de la chose qui pense, la philosophie n'a pas besoin de l'hypothèse d'une détermination de l'amour.

C'est une autre question de savoir jusqu' où Descartes restera fidèle à cette omission de 1641. Nous pourrions soutenir en effet que Descartes a eu une doctrine de l'amour ${ }^{5}$. Ou plutôt nous pourrions discuter quelle a été la centralité de cette doctrine. Je ne prends ici l'ajout de la traduction française au texte latin que comme un indice que le texte latin lui-même ne mentionnait pas l'amour et la haine comme l'une des modalités originelles de la res cogitans qui confirme

\footnotetext{
${ }^{3}$ Descartes, CEuvres, éd. Adam \& Tannery, tome VII (abrégé AT VIII), p. 34, lg. 118-21.

${ }^{4}$ Descartes, CEuvres, AT IX-1, p. 27.

${ }^{5}$ On trouve en effet, dans une lettre de 1645 ou 1646, cette note: «Ita amor, odium, affirmatio, dubitatio etc. sunt veri modi in mente... » (AT IV, p. 349, lg. 8-9).
} 
(comme chez Hegel) l'écart que la philosophie moderne tend à instaurer entre l'amour et son concept. Quand je dis la philosophie moderne, j'entends bien sûr aussi l'ensemble des sciences humaines développées par la pensée strictement contemporaine. Car le discours le plus commun sur la question de l'amour y revient la plupart du temps à considérer que ce que nous appelons l'amour - cet ensemble d'émotions, de passions et de pulsions - se résume en une conséquence, transcrite dans l'indétermination de la représentation psychologique, de jeux de déterminismes qui relèvent de la biologie, de la chimie, de la physique, bref dans un ensemble de déterminations qui trouvent leur vérité dans les enquêtes et formulations proprement scientifiques. Nous sommes en effet prêts à admettre que l'amour se résume dans l'effet non identifié de nécessités dont le concept n'apparaît jamais clairement à celui qui éprouve l'amour. L'amour, en quelque manière, devient le résultat inadéquatement connu de processus qui, eux-mêmes, ne relèvent pas de l'érotique. C'est devant cette situation que, je pense, la philosophie se trouve prise en défaut, prise en défaut par le fait qu'elle ne peut penser l'amour qu'en le réduisant à un autre que lui-même.

Mais cette entreprise peut pourtant s'inverser. Et c'est ce que je voudrais rapidement indiquer en essayant de montrer comment pourrait se substituer à la réduction du phénomène de l'amour un concept qui, lui-même, ne relève pas de l'érotique, voire comment s'établirait une réduction de l'ensemble de l'expérience par le phénomène amoureux lui-même. Passer d'une réduction de l'amour à une réduction par l'amour, une réduction érotique.

Pour ce faire, nous procèderons en plusieurs temps. Premièrement, tentons de montrer comment la question de l'amour peut devenir une question théorique pourvu qu'on prenne au sérieux la détermination contemporaine du nihilisme. Ensuite j'essaierai de montrer comment la réduction érotique s'exerce elle-même dans l'espace, dans le temps et dans la définition de l'ipséité. Premier point : pourquoi la question érotique peut-elle jouer un rôle conceptuel et théorique ? Pourquoi la question de savoir si je suis aimé, la question «m'aime-t-on d'ailleurs ? », dépasse-t-elle l'expérience propre de tel ou tel individu dans sa subjectivité anecdotique et inessentielle? Pourquoi cette question peut-elle endosser le poids de l'universel ? La réponse tient à ce que l'on peut thématiser sous le titre du nihilisme. Qu'est-ce que le nihilisme ? On désigne sous ce titre, depuis au moins Nietzsche, l'époque de la pensée rationnelle où les plus hautes valeurs se dévalorisent. Ceci signifie que tout énoncé, autrement dit tout énoncé certain se trouve soumis à l'interrogation qui demande, devant cette certitude 
même «à quoi bon? ». Pour entendre ce qu'elle signifie, il suffit de songer à l'objection de Pascal à l'encontre de Descartes : "Descartes inutile et incertain $»^{6}$. Car, à l'origine, la copie écrite disait plutôt «Descartes inutile et certain $»^{7}$, comme si la certitude - et non pas l'incertitude, ce qui resterait banal et presque trivial - elle-même se révélait inutile. Il s'agit, dans le cas de cette formulation rectifiée, d'une formulation strictement nihiliste, d'une définition du nihilisme : même et surtout la certitude ne sert de rien face au soupçon de sa dévalorisation : «à quoi bon ? ». Le nihilisme ne met en effet pas en cause la vérité des énoncés scientifiques ou philosophiques; il les surdétermine et les disqualifie en les condamnant pour inutilité. " À quoi bon? », en allemand «Umsonst», disqualifie au motif d'inutilité, et donc de vanité, ce qui est certain. Les transcendantaux du vrai, $\mathrm{du}$ bon, du bien, de l'être, ne sont pas réfutés parce qu'ils ne seraient pas véritablement ce qu'ils énoncent, ni parce qu'ils devraient recevoir une fondation plus radicale par une instance plus transcendante et plus certaine encore ; ils sont disqualifiés en tant même que bons, en tant que biens, en tant qu'étants. Ce qui disqualifie les transcendantaux ne tient ni à ce qu'ils sont, ni aux limites de ce qu'ils sont, ni aux conditions de leur validité, mais au simple fait que, à propos de chacun d'eux, on peut demander toujours «à quoi bon?". A quoi bon être plutôt que n'être pas, à quoi bon le bien plutôt que le mal ? La réponse à ces questions, pour Nietzsche, ouvre en effet elle-même un chemin de renvoi à une autre instance, autrement menaçante, par exemple le renvoi de la vérité à la volonté de vérité. Car la vérité de la vérité signifie que la vérité reste un simple effet de la volonté de puissance. Nous ne recherchons jamais la vérité pour elle-même, mais comme l'un des visages possibles, l'un des outils disponibles et l'une des armes disponibles de la volonté plus radicale de la puissance ellemême. Il s'agit de comprendre la vérité à partir de la volonté en elle, de la volonté qui ne veut par elle que son propre accroissement, sa montée en puissance, son passage à la puissance. La vérité doit, pour se comprendre, se réduire à la volonté de puissance. Tel est le nihilisme, quand il pose la question « À quoi bon?».

Or cette question, et son universalisation dans le nihilisme global, paradoxalement rend à la question érotique sa force et son universalité. Car à la question «à quoi bon? » ne peut répondre - telle

\footnotetext{
${ }^{6}$ Pascal, Pensées, Paris, éd. L. Lafuma, Pensées sur la religion et quelques autres sujets, Paris, Editions du Luxembourg, 1954, §887.

${ }^{7}$ Littéralement «Descartes inutile et certenne», Copie 9.203, Paris, éd. L. Lafuma, Pensées sur la religion et quelques autres sujets, Paris, Editions du Luxembourg, 1954, t.1, p. 472.
} 
serait $\mathrm{du}$ moins mon hypothèse - que ce qui répond à une autre question, la question " $\mathrm{m}^{\prime}$ aime-t-on ? ». La réponse à cette question, réponse encore indéterminée, constitue la seule réponse qui permette de résister à l'interrogation radicale imposée par le soupçon nihiliste. «À quoi bon? " ne peut pas recevoir de réponse ni recevoir la moindre résistance par un surcroît de certitude théorique, car la certitude dans la théorie reste elle-même par excellence susceptible de tomber sous le coup du soupçon nihiliste. Au contraire, le fait de dire (croire ou constater, ou croire constater) que «l'on m'aime d'ailleurs » suffit (seul) à établir une réponse et dresser une résistance à la question « à quoi bon? ». L'affirmation que «l'on m'aime d'ailleurs » instaure en effet une instance externe antérieure à la certitude interne elle-même, à savoir précisément qu'un autre que moi m'aime d'ailleurs. Antérieure à la certitude elle-même, antérieure au cercle des transcendantaux se convertissant l'un dans l'autre, antérieure à l'illusion ou pire à la réalité de l'autonomie cerclée. La certitude se retrouve toujours disqualifiée par la question «à quoi bon? », mais cette question ne peut disqualifier le fait que, quoi qu'il arrive, je me trouve aimé d'ailleurs. Je ne peux jamais répondre à l'interrogation «à quoi bon faire ceci, à quoi bon être soi-même, à quoi bon vivre ? » en disant "parce que je le veux", mais seulement en constatant, sans aucun pourquoi, «qu'un autre le veut pour moi». Cette réponse "qu'un autre m'aime » apparaît la seule qui me permette de ne pas sombrer devant $\mathrm{l}^{\prime}$ " à quoi bon ? » du nihilisme.

On peut le redire autrement, en imaginant une contre-épreuve à la question posée par Méphisto à Faust. Soit le contrat fantasmé où j'aurais à choisir entre rester ce que je suis, mortel, ou bien, en échange de mon âme, recevoir la possession de toutes les puissances et richesses du monde. Transposons ce marché supposé dans cet autre contrat où l'on m'offrirait non pas de donner mon âme (car je n'en ai peut-être pas et mon interlocuteur n'aurait peut-être aucune envie de s'en approprier les irréalités), mais d'abandonner simplement, en échange d'une vie aussi longue que je voudrais, avec toute la puissance et toute la richesse de la terre, la possibilité pour moi d'aimer ou d'être aimé. Bref acheter tout au prix de la possibilité d'aimer et de me faire aimer. Qui accepterait ? Renoncer à la possibilité, ne fût-ce que la possibilité, d'aimer ou d'être aimé, pour en échange vivre une éternité de temps, de jouissance et de pouvoir? Il n'est pas certain qu'aucun d'entre nous n'accepte ce marché, qui implique une manière de castration transcendantale par quoi je devrais vivre une éternité sans l'amour - et peut-être même que n'auraije pas même le courage de vivre cette éternité, puisque, comme le dit 
Woody Allen, l'éternité, c'est très long, surtout vers la fin, surtout une éternité à aucun moment érotisable, sans que la possibilité de son érotisation n'en soit maintenue. Est-ce qu'une telle vie serait encore humaine, ou animale, ou angélique, ou simplement robotique ? La possibilité $d$ 'une vie sans aucun phénomène érotique peut-elle même s'envisager et se concevoir?

D'une certaine manière, en posant la question ainsi, on comprend comment le nihilisme doit se trouver confronté à rien de moins qu'à la question érotique, et pourquoi celle-ci peut trouver une pertinence universelle. L'instance érotique n'y reste plus simplement subjective, close dans la sphère individuelle, préconceptuelle ; elle surgit radicale, universelle et rationnelle. Elle le devient aussitôt qu'elle s'affronte à l'interrogation nihiliste, où elle prend rang d'une opération théorique. L'instance érotique, la question qui demande " $\mathrm{m}^{\prime}$ aime-t-on d'ailleurs? » opère en effet, ainsi vue, une réduction, qui devient visible tout naturellement pourvu qu'on la confronte à cette autre réduction qu'est le nihilisme. Comment donc opère la réduction érotique? Il y a un principe de phénoménologie évident, que tout le monde connaît, mais qu'il vaut de rappeler : il ne peut pas y avoir de phénomène (ni de phénoménologie) que l'on puisse décrire, donc voir, si l'on ne procède pas à une réduction. La réduction consiste à tenter de déterminer ce qui se trouve effectivement donné à la conscience. Aucun phénomène ne peut se déployer sans, d'abord, s'assurer un vécu de conscience. La grande difficulté vient alors de savoir ce que nous ignorons dans l'attitude naturelle - l'ampleur et les limites du donné. Il faut donc, pour dégager les limites du donné, c'est-à-dire de ce à partir de quoi un phénomène peut se constituer, soumettre le donné supposé sans critique à une réduction critique. Comment le phénomène érotique, s'il y en a un, satisfait-il donc à la logique de la réduction ? Quelle réduction permet le phénomène érotique? Bref, y-a-t-il une réduction érotique ? Cette réduction érotique, on peut de fait la décrire. Esquissons-la rapidement.

La réduction érotique joue d'abord évidemment dans les deux dimensions du donné, c'est-à-dire, pour parler comme Kant, dans la dimension d'abord de l'intuition, puisque l'intuition seule est donatrice. Le donné se joue donc dans les formes de l'intuition, dans l'espace et dans le temps. - Comment la réduction érotique s'exerce-telle dans l'espace ? Soit la situation érotique élémentaire qui se décrit dans la formulation de la première question « $\mathrm{m}^{\prime}$ aime-t-on d'ailleurs ? », en d'autres termes, la situation érotique dans laquelle je suis amoureux. Comment cette situation érotique opère-t-elle une réduction de l'espace? Le point tout à fait essentiel tient à ce que, dans 
l'espace érotique, je ne suis jamais à l'endroit où je suis, au contraire de la situation non érotique, où, dans l'espace commun, je définis le centre parce que je l'occupe. En situation érotique, dans l'espace, je suis toujours et essentiellement décentré. Dans l'espace de l'attitude naturelle, qui est en même temps l'espace de l'expérience quotidienne et de l'expérience scientifique, je suis au centre de l'espace empirique. Je suis à titre d'observateur privilégié celui à partir duquel l'espace se déploie et se mesure. Mais dans la situation de réduction érotique, la réduction de l'espace fait que je ne suis jamais au centre : au centre de cet espace se trouve le centre érotique pour moi, qui par définition n'est pas moi. Pour moi, le centre de l'espace érotique désigne toujours celui dont je suis amoureux. Il y a donc un décentrement fondamental de l'espace, qui fait que la description de l'espace phénoménologique que Husserl élabore, par exemple, avec sa doctrine de l'intersubjectivité dans la Méditation cartésienne $V$, se trouve en quelque manière contredit. Husserl explique, ce qui semble aller de soi, que, dans l'intersubjectivité habituelle pour lui non érotique, je suis toujours ici et l'autre toujours là-bas. Je pourrais certes, par une variation imaginative, me mettre au point de vue de l'autre, c'est-àdire me mettre là-bas et imaginer que lui est ici; mais quand je renverse l'écart, bien entendu, il en reste toujours un, où le je, quel qu'il soit, a encore en propre d'être ici, tandis qu'un autrui, quel qu'il soit, a en propre d'être là-bas. Je peux imaginer que c'est autrui qui tient le rôle de je, et moi qui tient le rôle d'autrui, mais dans ce cas, nous échangeons seulement les déterminations spatiales, sans les détruire. Tel n'est pas le cas dans la réduction érotique. Là, le je, à savoir moi, est toujours là-bas. Et autrui est toujours ici. Donc il me revient à moi de dire que, essentiellement, «je ne suis pas là où il est ». C'est toujours moi qui suis là-bas; ce n'est pas l'autre qui est absent, mais c'est moi qui ne suis pas là où je devrais être. Je perçois toujours l'espace érotique à partir d'un centre que je n'occupe jamais. L'espace érotique a toujours un centre où je ne suis jamais et une périphérie où je suis toujours, parce qu'il se définit à partir d'autrui que je ne serai jamais. Il suffit que l'autrui privilégié se déplace pour que le centre se déplace aussi pour moi, et je suis plus ou moins proche de l'autre, je suis plus ou moins ailleurs et parti selon ma distance par rapport au centre, que je ne suis jamais. Il ne suffit plus, comme dans l'attitude naturelle (non érotique) que je sois quelque part pour être au centre, car, si dans l'expérience empirique, non érotique et "normale », le centre, reste l'endroit où je suis, dans la situation érotique, autrui possède le centre et le déplace avec lui. Et c'est pourquoi, moi qui ne suis pas au centre, même si je suis en 
(peut-être joyeuse) compagnie, je peux pourtant dire que là où je suis, il n'y a, ou n'y avait, personne. Et ceci non pas parce que j'étais seul comparé à d'autres individus empiriques, mais parce que le centre ne se trouvait pas au milieu de la foule qui m'entourait. Le centre manquait, et tout était dépeuplé. Donc quand je dis : il n'y avait personne parce qu'il ou elle n'était pas là, je définis bien le fait qu'il y ait quelqu'un dans un centre, où je ne me trouve jamais. Il y a donc bien un espace érotique dont la règle fondamentale édicte que je n'en constitue jamais le centre et je n'occupe jamais le «ici » de cet espace érotique.

Nous pouvons répéter la même analyse si nous nous situons dans le temps érotique. Le temps érotique déroule un flux dans lequel je peux parfaitement dire qu'au milieu des mille occupations d'un emploi du temps très chargé, il ne se passe rien. Pendant un laps de temps d'un jour, d'un mois, d'un an, il ne s'est rien passé. C'est entièrement faux pour l'attitude naturelle non érotique, car bien entendu la vie a continué à se passer : j'ai continué à dormir, à voir des gens, à me nourrir, etc., et pourtant je dis justement qu'il ne s'est rien passé. Car il y a un ennui érotique. Ce qui ne veut pas dire que je ne fais rien et que j'ai du temps pour m'ennuyer, mais veut dire que rien n'arrive. Que rien n'arrive depuis le centre érotique. Et de la même manière que je ne suis jamais au centre de l'espace érotique mais toujours à sa périphérie, il ne suffit pas que je sois dans l'instant érotiquement présent pour qu'il se passe vraiment quelque chose. Il ne suffit pas que le monde continue à se dérouler pour que je me trouve en une situation où arrive un événement érotique, qui fait qu'il se passe quelque chose, c'est-à-dire qu'il y ait du présent. Pour qu'il y ait du présent, il faut que le présent me fasse le cadeau de la présence de celui qui, ou celle qui, constitue l'origine du flux érotique. À ce moment-là, dans ce flux érotique, le temps devient présent. Il faut qu'il ou elle m'appelle, vienne, surgisse, arrive, bref se manifeste et qu'il intervienne dans mon temps. Alors seulement, je pourrais dire que tout ce qui advient sans signification érotique se trouve annulé et qu'il se passe quelque chose : il ne se passera que ce qui n'est plus annulé érotiquement, à savoir l'événement de celui ou celle qui ouvre mon flux temporel érotique. Et donc le passé ou le futur se détermineront par rapport à cet événement érotique. C'est ainsi par exemple que je comprendrai la nécessité de la répétition du discours érotique. Supposons que je sois distinct de celui ou celle qui peut répondre à la question " $\mathrm{m}$ 'aime-t-on? », celui dont nous dirons vulgairement que je suis amoureux. Il ne se passe quelque chose que si celui-ci me dit «je t'aime ». Et le présent ne se trouve accompli qu'à 
chaque fois qu'il me dit «je t'aime». Mais comme, par définition, le «je t'aime» ne dure pas, il faut donc que le présent se répète, et le présent se répète à chaque «je t’aime » qui est répété. Le temps luimême se répète par l'événement érotique fondamental qui consiste à dire ou à entendre " je t'aime ». Donc je peux parfaitement dire « il ne s'est rien passé », bien que tout se passe autour de moi, parce que le « je t'aime » n'a pas été performé. Et il se passera quelque chose aussi souvent et aussi longtemps que le « je t'aime » sera performé.

Nous avons un cas tout à fait caractéristique de réduction, puisqu'ici le présent ne dépend pas de la succession psychologique du flux des vécus, mais il dépend de l'intervention dans le flux du vécu d'un événement qui ne subsiste pas à ma disposition, ni d'ailleurs à la disposition du flux lui-même, car il provient de celui qui joue, dans ce flux, le rôle de centre érotique dans l'espace. Il y a donc une réduction de l'espace par l'ailleurs érotique; et il y a une réduction du temps par le maintenant érotique. Dans les deux cas, l'espace et le temps de l'attitude naturelle se trouvent annulés. L'espace galiléen, ou newtonien, est suspendu, et le temps du flux est lui aussi considéré comme ne se passant pas puisqu'il n'est pas scandé par le « je t'aime ». - Bien entendu il est possible de poursuivre cette réduction en la faisant porter sur l'ipséité. Car il y a une réduction de l'ipséité, de l'identité du je (éventuellement de son identité à lui-même) dans sa singularité absolue, son haeccitas, par la réduction érotique. En effet, comment puis-je définir ce qui en moi serait absolument moi ? Je ne peux le définir qu'en écartant tout ce qui en moi peut advenir par quiconque d'autre que moi. On peut envisager ici la thématique du sujet transcendantal - qui n'est aucun d'entre nous et peut être mis en œuvre par n'importe lequel d'entre nous, dans les conditions, dirait Husserl, de la normalité. Mais on peut dire aussi que nous avons affaire ici au je de la pensée, à l'entendement agent universel, et ce je de la pensée peut s'accomplir en n'importe lequel d'entre nous. N'importe lequel d'entre nous peut vérifier que «ego sum, ego existo", chaque fois que je performe cet énoncé dans ma pensée. Nous pouvons aussi penser à ce je quiconque et quelconque sous le titre de ce que Heidegger appelait le On (das Man), c'est-à-dire celui qui peut être remplacé par n'importe qui. En effet, dans les règles de la vie sociale et de sa fonctionnalité (je crois que chez les gens qui gèrent les ressources humaines, c'est-à-dire qui mettent en œuvre le chômage, on parle d'employabilité), l'employabilité indique très bien que chaque individu peut être remplacé par n'importe quel autre à la mesure de l'universelle norme de l'employabilité : celle-ci veut donc bien dire, dans sa violence, qu'il s'agit pour chacun de se faire à son 
emploi, au sens presque théâtral. Cet emploi devient le nom commun d'un nombre indéfini d'individus. Il y a donc des emplois qui font que chacun peut être substitué à n'importe qui et réciproquement. Ce que quiconque peut faire à ma place n'est justement pas ce qui définit mon ipséité ou mon haeccéité absolue. Inversement, qu'est-ce que personne ne peut faire à ma place ? Vivre, penser, sans doute d'autres peuvent-ils le faire à ma place. Après tout, penser, toute chose pensante peut le faire à ma place et penser les mêmes choses. L'une des règles fondamentales de l'objectivité édicte d'ailleurs la possibilité pour des individus différents de faire la même opération de pensée, et le fait que ces mêmes opérations de pensée, même subjectivement ordonnées de manière différente, arrivent au même résultat, permet de vérifier la vérité de ce qui est pensé. Donc l'interprétation intersubjective de la vérification présuppose que chacun peut penser tout ce que tout le monde pense. Par conséquent, penser ne $\mathrm{m}^{\prime}$ individualise précisément pas. Quoi donc m'individualise? Comment se manifeste le plus propre en moi ? Sans doute, par le vouloir, l'usage de mon libre-arbitre. Et éventuellement le bon usage de mon librearbitre : "Le libre arbitre est de soi la chose la plus noble qui puisse être en nous [...] le plus grand de tous nos biens [...] ; il est aussi celui qui est le plus proprement nôtre $»^{8}$. Mais mon libre-arbitre s'avère-t-il toujours proprement mien du simple fait que c'est moi qui veux, et qu'un autre pourrait vouloir autrement? Tout dépend de ce que le libre-arbitre lui-même veut. Il y a sans doute une forme de la volonté qui m'identifie, c'est la volonté en tant qu'elle permet d'aimer, c'est-àdire de m'unir de volonté à ce qui m'apparaît comme un bien. Sans doute aimer ou me faire aimer par un autre définit-il mon propre. Et cela se vérifie par le fait que (du moins par hypothèse) nul ne peut aimer à la fois deux personnes sous le même rapport et au même moment. Donc l'individuation résulte d'une réduction érotique. La réduction érotique permet précisément d'individualiser celui que l'on aime, qu'il le veuille ou non, que ce soit pour son bien ou pour son malheur. La réduction érotique permet à l'amant, par cristallisation, par destin, par mauvaise habitude, par arbitraire, d'identifier à luimême celui qu'il aime. Le propre de la situation érotique tient à ce que je ne peux pas, dans la posture érotique stricte, je ne peux pas me tromper sur celui que j'aime. Il est celui que j'ai identifié, pour de bonnes ou de mauvaises raisons, peu importe, l'unique, dégagé par la

\footnotetext{
${ }^{8}$ Descartes, Lettre à Christine, 20 novembre 1647, AT V, p. 85. Voir également : « ...il n'y a rien qui véritablement lui [sc. le généreux] appartienne que cette libre disposition de ses volontés » (Passions de l'âme, §93).
} 
situation érotique et qui, en retour, m'identifie. C'est l'identification réciproque de l'un à l'autre, c'est-à-dire l'individuation, qui caractérise le phénomène érotique. On peut se demander $\mathrm{d}$ 'ailleurs si le problème classique de l'individuation - $\mathrm{y}$ a-t-il une individuation par la matière, par la forme, etc. - ne conduit pas, au bout du compte, à ce résultat que l'individuation est érotique ou n'est pas.

Dès lors, nous aurions atteint les trois dimensions d'une réduction érotique. L'amour ici produit comme un concept une détermination de l'intuition dans l'espace, dans le temps et une détermination du concept de celui qui aime et de celui qu'il aime dans la problématique de l'individuation. Ce que je soutiens et qui peut être discuté, tient en ceci que, loin que l'amour reste l'effet non conceptuel d'une détermination conceptuelle étrangère, et loin que l'amour soit réduit par un autre concept, il devient lui-même ce qui opère la réduction de l'ensemble des dimensions de l'expérience. Pour dire ceci, il faut supposer que l'amour, comme disposition érotique, puisse déterminer la totalité de l'expérience. Ceci a-t-il un sens ? Cela a sûrement un sens si nous prenons au sérieux une thèse qui a été pour la première fois posée, non par Heidegger, comme celui-ci le laisse parfois supposer, mais par saint Augustin. Cette thèse dit que l'amour ne se résume ni à un sentiment, ni à une passion, mais constitue une détermination permanente de l'ego. Il n'y a pas d'ego qui ne se trouve pas dans une situation déjà érotique. C'est ce qu'indique clairement un célèbre passage de saint Augustin : "Nemo est qui non amet. Sed quaeritur quid amet. Non ergo admonemur ut non amemus, sed ut eligamus quid amemus $»^{9}$. Autrement dit, il n'y a personne qui n'aime pas; à chacun, l'on ne demandera donc pas s'il aime, mais seulement ce qu'il aime. La question demande de choisir ce que nous aimons, non pas de savoir si nous aimons, ou non, car nous ne pouvons pas ne pas aimer. L'amour n'a rien d'une passion, mais ouvre l'horizon de la subjectivité elle-même. Donc, si la question ne veut pas savoir si nous aimons ou si nous n'aimons pas, puisque nous nous trouvons toujours en situation d'aimer, mais veut savoir ce que nous aimons, et comment, alors la réduction érotique s'avère toujours au moins latente. L'expérience pour nous s'organise par une réduction qui ouvre le champ du donné selon des limites érotiques. En d'autres termes, notre expérience est aussi large que notre capacité à

\footnotetext{
9 Sermon 34, 2, P.L. 38, col. 210. Voir Hugues de Saint-Victor: «Vita tua dilectio est, et scio quod sine dilectione esse non potes » (De Arrha Animae, 25-26, Opera omnia, Brepols, Turnhout, 1997, p. 226; et Thomas d'Aquin: "Respondeo dicendum quod nulla alia passio est, quae non praesupponat aliquem amorem. Cujus ratio et quia omnis alia passio animae importat motum ad aliquid, vel quietem in alio » (Summa theologiae Ia IIae, q. 27, a.4, resp.i).
} 
entrer en situation érotique. Nous ne vivons que ce que nous envisageons comme une expérience possible, dont les limites se trouvent fixées par la réduction érotique. Plus nous aimons, plus nous ouvrons le possible. Plus nous restreignons la réduction érotique, plus le champ de l'expérience possible se trouve lui-même déterminé. Heidegger à sa façon, expressément en le reprenant de saint Augustin ${ }^{10}$, avait posé la thèse que le Dasein n'était jamais sans une détermination, une tonalité affective (Stimmung). Il n'y a jamais de Dasein neutre, puisqu'il se trouve toujours déjà orienté vers le monde à la mesure de sa préoccupation. De la même manière que Heidegger ne peut guère interpréter la situation amoureuse que comme tonalité affective, nous pouvons prétendre que la tonalité affective par excellence revient évidemment à l'amour. L'amour constitue la tonalité affective fondamentale, et dans la mesure où je suis en réduction érotique, un monde s'ouvre pour moi. Ici joue à plein la formule de saint Augustin: "Non intratur in veritatem nisi per caritatem ${ }^{11}$. Proposition épistémologiquement presque évidente : on n'entre dans la vérité que par la charité veut dire que nous n'entrons dans l'expérience du monde qu'à la mesure de la réduction érotique qui nous l'ouvre.

Ainsi posée la question du phénomène érotique change de nature : elle devient une question théorétique et non pas une simple question pratique, morale, ni éthique, ni évidemment religieuse ou ni érotique au sens étroit de ces termes. Mais une question théorétique à la mesure de la réduction érotique par quoi l'expérience mondaine se détermine et se délimite. Dans ce contexte où l'espace, le temps et l'individuation résultent de la situation érotique et non pas l'inverse, la question de la réduction érotique et du phénomène érotique ne désignent plus une région particulière de l'expérience, mais le lieu d'une phénoménologie, y compris au sens hégélien du terme, c'est-àdire une description des figures de la conscience. La situation érotique produit les figures de la conscience. Ou, dit autrement, il faut concevoir l'«...amour en tant que le motif fondamental du comprendre phénoménologique $»^{12}$. Ces figures, on se bornera ici à les énumérer ;

\footnotetext{
${ }^{10}$ Le cours Phänomenologie des religiösen Lebens (1921, G.A. 60, p. 204) cite le Sermon 198, 2 (P.L. 38, col. 1024) : "Nemo quippe vivit in quacumque vita, sine tribus istis animae affectionibus, credendi [vertrauend zugreifen, irgendwie ein Ende festmachen], sperandi [erwartend, sich offenhalten für], amandi [liebende Hingabe, für wert halten] ».

${ }_{11}$ Contra Faustum, 32,18, P.L. 42, col. 507. Cité et commenté par Heidegger, Sein und Zeit, §29, p. 139 , note 1 .

12 Heidegger: «Liebe als Grundmotiv ders phänomenologischen Verstehens » (GA 58, p. 185). $\mathrm{Ou}$ : «In der Liebe ist Verstehen » (ibid., p. 168).
} 
elles dépendent de trois questions successives qui scandent l'odyssée de la conscience érotique : la première question, demande « m'aime-ton, d'ailleurs? »; la seconde question, qui intervient parce que la première ne peut trouver dans ses termes de réponse, demande " puis-je moi-même aimer le premier ? "; et la troisième question, qui répond à la deuxième question consiste en fait en une constatation : «j'ai toujours déjà été aimé avant d'avoir aimé ». Tels apparaissent les trois moments de la réduction érotique, qui permettent de dégager les figures de la conscience, et parmi ces figures, la plus fondamentale l'interprétation de l'ego lui-même, non pas seulement comme pensant, mais bien comme aimant. C'est-à-dire l'amant, l'ego amans, comme on dit la res cogitans. C'est à partir de l'amant ainsi défini que se met en place la logique de la conscience érotique, à savoir qu'elle aime la première. Le propre de la conscience érotique, imitant et réfutant à la fois la spontanéité de l'aperception transcendantale, revient toujours à ceci, que l'amant - dans l'avance - aime le premier. Il anticipe et cette anticipation même le constitue comme l'amant, celui qui aime sans savoir si on l'aime en retour. 